\title{
Diagnosis of Pseudoexfoliation Syndrome in Pseudophakic Patients
}

\author{
Amir Sternfeld ${ }^{a, b} \quad$ Moshe Luski ${ }^{a, b}$ Ruti Sella ${ }^{a, b} \quad$ Alon Zahavi $^{a, b} \quad$ Noa Geffen ${ }^{a, b}$ \\ Avihu Pereg $^{c}$ Elinor Megiddo ${ }^{a, b}$ Dan Gaton ${ }^{a, b}$ \\ ${ }^{a}$ Department of Ophthalmology, Rabin Medical Center, Beilinson Hospital, Petach Tikva, Israel; ${ }^{b}$ Affiliated to \\ Sackler Faculty of Medicine, Tel Aviv University, Tel Aviv, Israel; ' $F$ Faculty of Health Science, Ben Gurion University, \\ Beer-Sheva, Israel
}

\section{Keywords}

Pseudoexfoliation syndrome · Pseudophakia · Glaucoma ·

Cataract $\cdot$ PES glaucoma

\begin{abstract}
Objective: The aim of this study was to evaluate the sensitivity and specificity of pseudoexfoliation syndrome diagnosis in pseudophakic patients and potential means of improving it. Methods: This prospective, nonrandomized study comprised 41 consecutive patients (41 eyes) scheduled for cataract surgery at a tertiary medical center during 2016 and 2017. Preoperatively, all patients underwent a detailed slitlamp examination, including gonioscopic assessment of the iridocorneal angle. The examination was performed by a glaucoma specialist who completed an assessment form documenting the presence/absence of clinical signs of pseudoexfoliation syndrome. It was repeated 1-2 weeks postoperatively by a second, masked, glaucoma specialist. Results: Sixteen patients (39.0\%) were diagnosed with pseudoexfoliation syndrome preoperatively. The diagnosis was confirmed postoperatively in $11 / 16$ patients ( $68.8 \%$ sensitivity) and in an additional patient not diagnosed preoperatively (96\% specificity). The ability to diagnose pseudoexfoliation syndrome postoperatively was significantly worse than pre-
\end{abstract}

operatively $(Z=12.161, p<0.0001)$. Pupillary border deposits (75\% of cases) and the Sampaolesi line (83.3\%) were the cornerstones of the postoperative diagnosis; anterior capsular deposits were evident in only $41.6 \%$ of cases diagnosed postoperatively (31.3\% of the originally diagnosed cases). Conclusions: Underdiagnosis of pseudoexfoliation syndrome is common in pseudophakic patients and may have significant implications for future management. Careful attention to pupillary border anatomy and meticulous gonioscopic assessment of the iridocorneal angle are essential for accurate diagnosis. Preoperative documentation of pseudoexfoliation syndrome could help prevent this diagnostic pitfall.

(c) 2020 S. Karger AG, Basel

\section{Introduction}

Pseudoexfoliation syndrome (PES) is a common systemic disorder affecting >60 million people worldwide [1]. It is characterized by the production and deposition of an extracellular fibrillary material in tissues throughout the body [2]. Ocular findings include small white deposits of the fibrillar material on several anterior segment structures, specifically and most frequently the anterior karger@karger.com

www.karger.com/ore

(C) 2020 S. Karger AG, Base

Karger"
Amir Sternfeld

Department of Ophthalmology

Rabin Medical Center, Beilinson Hospital

IL-4941492 Petach Tikva (Israel)

shtery@gmail.com 
lens capsule and pupillary border [3]. Less frequently, the deposits are seen on the corneal endothelium, iridocorneal angle, ciliary processes, and zonules [3]. Another prominent ocular finding is pigment loss from the iris sphincter region causing transillumination defects and loss of the pupillary ruff. The pigment accumulates on the trabecular meshwork and anterior to Schwalbe's line as a wavy pigmented line (Sampaolesi line) [1-3].

The incidence of PES increases with age, and the prevalence varies considerably worldwide. Nordic countries are reported to have the highest prevalence. A study from Sweden of the population aged over 66 years reported a mean prevalence of $23 \%$, rising to $61 \%$ in the age group over 87 years [4]. By contrast, in Australia, McCarty and Taylor [5] noted a prevalence rate of only $1 \%$.

The diagnosis of PES has significant implications for clinical management, as PES is a major risk factor for elevated intraocular pressure and glaucoma. About half of all patients with PES will have glaucoma at some point in their lives, making PES the most common cause of secondary open-angle glaucoma worldwide, and in some countries of all open-angle glaucoma in general [1]. Furthermore, in patients with PES, ocular hypertension $(\mathrm{OHT})$ is twice as likely to convert to glaucoma $[3,6]$. PES-related glaucoma poses a greater treatment challenge than primary open-angle glaucoma because patients present with a higher mean intraocular pressure and have significant daily intraocular pressure fluctuations, worse optic neuropathy at diagnosis, and a worse clinical course. They are also less responsive to medical therapy, leading to a higher rate of surgical interventions. Therefore, patients with PES require closer lifelong follow-up and medical attention than some patients with other types of glaucoma [1-3,7-11].

PES is associated with higher than normal rates of postcataract-surgery subluxation or dislocation of the implanted intraocular lens because of weakness and impaired anchoring of the zonules to their origin and insertion sites $[12,13]$. A preoperative diagnosis of PES should raise the index of suspicion for this complication, facilitating its postoperative detection and treatment.

The diagnosis of PES is particularly challenging in the pseudophakic population because the circular portion of the anterior lens capsule that is removed during capsulorhexis often displays pseudoexfoliative fibrillary deposits that facilitate the diagnosis [3].

The primary aim of this study was to evaluate the detection accuracy of PES in pseudophakic patients. Our secondary aim was to identify those clinical signs that facilitated PES diagnosis in the pseudophakic population.

Pseudoexfoliation Syndrome in

Pseudophakic Patients

\section{Subjects and Methods}

A prospective nonrandomized design was used. The cohort included consecutive patients who were admitted for same-day cataract surgery to the ophthalmology department of a single tertiary medical center during 2016 and 2017. The inclusion criteria were age 18 years or above, the ability to understand and sign an informed consent form, and willingness and ability to attend the follow-up visit. Exclusion criteria were any prior intraocular surgery, prior diagnosis of closed angle glaucoma, and systemic or ocular pathologies preventing detailed slit-lamp examination and PES diagnosis.

\section{Data Collection and Follow-Up Examination}

Preoperatively, all patients underwent a detailed slit-lamp examination including assessment of the iridocorneal angle using a gonioscope (Zeiss 4 Mirror Gonioscopy Lens, Carl Zeiss AG, Oberkochen, Germany) before and after pupillary dilation by a glaucoma specialist. The findings were entered into an assessment form documenting the presence or absence of clinical signs of PES: fibrillary deposits on the anterior capsule, pupillary border, iridocorneal angle, zonules or corneal endothelium, and Sampaolesi line. The examiner also documented optic nerve morphology and possible glaucomatous state. The patient was then classified as PES or non-PES.

This assessment was repeated 1-2 weeks after cataract surgery by a different glaucoma specialist who was masked to the results of the preoperative evaluation. The findings were entered into a follow-up assessment form including the same clinical parameters as the preoperative form. Neither examiner was allowed to assess the fellow eye for PES. Study participants who had an eventful/complicated cataract surgery or significant corneal edema on the postoperative follow-up examination were excluded from the statistical analysis because these factors could potentially affect the postoperative clinical evaluation for PES.

\section{Statistical Analysis}

Owing to the dichotomic nature of the parameters, $\chi^{2}$ test was applied to compare findings between examiners (first and second). The specificity and sensitivity of the postoperative diagnosis of PES and clinical signs of PES were calculated. The postoperative diagnosis rate of PES was compared to the preoperative rate by means of a $Z$ test for proportion comparison.

\section{Results}

Fifty-two patients were recruited for the study. Nine failed to attend the follow-up visit, 1 had an eventful cataract surgery (preoperatively diagnosed without PES) and another had significant corneal edema (preoperatively diagnosed with PES). The remaining 41 patients (41 eyes) were included in the final statistical analysis.

The first examiner categorized 16 patients as PES (39.0\%) and 25 patients as non-PES. The demographic and baseline features of the patients by the presence or absence of preoperative PES are detailed in Table 1. There 
Table 1. Demographic and baseline characteristics of the PES and non-PES groups

\begin{tabular}{lccc}
\hline & Non-PES & PES & $p$ value \\
\hline Patients (eyes) & 25 & 16 & $>0.05^{\mathrm{a}}$ \\
Age, years & $72.80 \pm 6.53(61-83)$ & $72.20 \pm 5.09(60-80)$ & $>0.05^{\mathrm{b}}$ \\
Male/female, $n$ & $9 / 16$ & $7 / 9$ & $>0.05^{\mathrm{b}}$ \\
Right/left eye, $n$ & $8 / 8$ & $12 / 13$ & $>0.05^{\mathrm{b}}$ \\
Diagnosis of glaucoma & $0(0)$ & $2(12.5)$ & $>0.05^{\mathrm{b}}$ \\
Family history of glaucoma & $1(6.3)$ & $3(12)$ & $>0.05^{\mathrm{a}}$ \\
Preoperative BCVA (logMAR) & $0.44 \pm 0.30$ & $0.52 \pm 0.35$ & $<0.05^{\mathrm{b}}$ \\
Deposits on anterior capsule & $0(0)$ & $13(81.3)$ & $<0.05^{\mathrm{b}}$ \\
Deposits on pupillary border & $0(0)$ & $8(50)$ & $<0.05^{\mathrm{b}}$ \\
Sampaolesi line & $1(4)$ & $15(93.8)$ & $0(0)$ \\
Deposits on iridocorneal angle & $0(0)$ & $0(0)$ & $>0.05^{\mathrm{a}}$ \\
Deposits on zonules & $0(0)$ & $0(0)$ & $>0.05^{\mathrm{b}}$ \\
Deposits on endothelium & $0.36 \pm 0.13$ & $2(8)$ & \\
CDR & $2(13.3)$ & $2(8)$ & \\
Suspected to be glaucomatous & & & \\
\hline
\end{tabular}

Data are presented as $n(\%)$ or the mean $\pm \mathrm{SD}$ (range). ${ }^{\text {a Student } t} t$ test. ${ }^{\mathrm{b}} \chi^{2}$ test. BCVA, best corrected visual acuity; CDR, cup to disc ratio; logMAR, logarithm of minimum angle of resolution; PES, pseudoexfoliation syndrome.

Table 2. Findings on preoperative and postoperative examination for PES

\begin{tabular}{|c|c|c|c|c|}
\hline Clinical features & Preoperative & $\begin{array}{l}\text { Post- } \\
\text { operative }\end{array}$ & $\chi^{2}$ & $p$ value $^{\mathrm{a}}$ \\
\hline \multicolumn{5}{|l|}{ Diagnosis } \\
\hline PES & $16(39)$ & $12(29.3)$ & 19.76 & $<0.001$ \\
\hline No PES & $25(61)$ & $29(70.7)$ & & \\
\hline \multicolumn{5}{|c|}{ Deposits on anterior capsule } \\
\hline Yes & $13(31.7)$ & $5(12.2)$ & 6.133 & $>0.05$ \\
\hline No & $28(68.3)$ & $36(87.8)$ & & \\
\hline \multicolumn{5}{|c|}{ Deposits on pupillary border } \\
\hline Yes & $8(19.5)$ & $9(22)$ & 9.539 & $<0.005$ \\
\hline No & $33(80.5)$ & $32(78)$ & & \\
\hline \multicolumn{5}{|l|}{ Sampaolesi line } \\
\hline Yes & $16(39)$ & $10(24.4)$ & 9.332 & $<0.005$ \\
\hline No & $25(61)$ & $31(75.6)$ & & \\
\hline
\end{tabular}

Data are presented as $n(\%) .{ }^{\text {a }} \chi^{2}$ test. PES, pseudoexfoliation syndrome.

were no statistically significant differences between the 2 groups in any of the demographic parameters evaluated.

The preoperative diagnosis of PES in the 16 patients was based on findings of 3 clinical features: Sampaolesi line (93.8\% of cases), anterior capsular deposits (81.3\%), and pupillary border deposits (50\%). Of the 25 patients in the non-PES group, 1 had a single suggestive sign of a Sampaolesi line (4.0\%) which was not sufficient for a de-

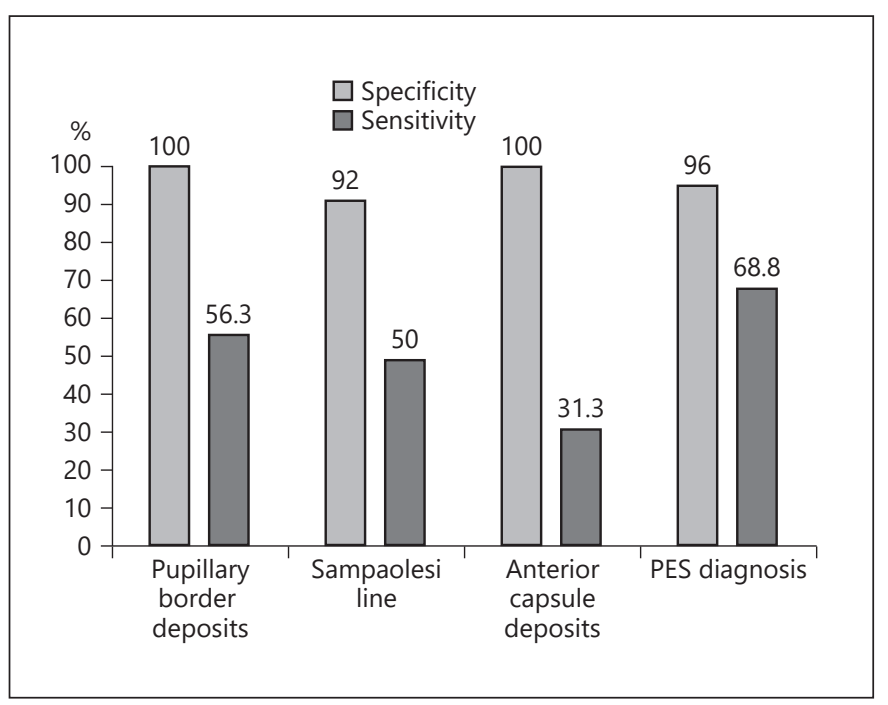

Fig. 1. Sensitivity and specificity of postoperative diagnosis of PES and of each major clinical sign separately for the diagnosis of PES.

finitive diagnosis. None of the examinations revealed deposits on the corneal endothelium, lens zonules, or iridocorneal angle.

Postoperatively, 12 patients were diagnosed with PES. They included 11 of the 16 patients with a preoperative diagnosis of PES (68.8\% sensitivity) and an additional patient from the preoperative non-PES group (96\% specificity). The ability to diagnose PES postoperatively was sig-
30

Ophthalmic Res 2021;64:28-33

DOI: $10.1159 / 000508336$
Sternfeld/Luski/Sella/Zahavi/Geffen/ Pereg/Megiddo/Gaton 
Fig. 2. Distribution of patients diagnosed with PES by the number of criteria found on their examination preoperatively and postoperatively.

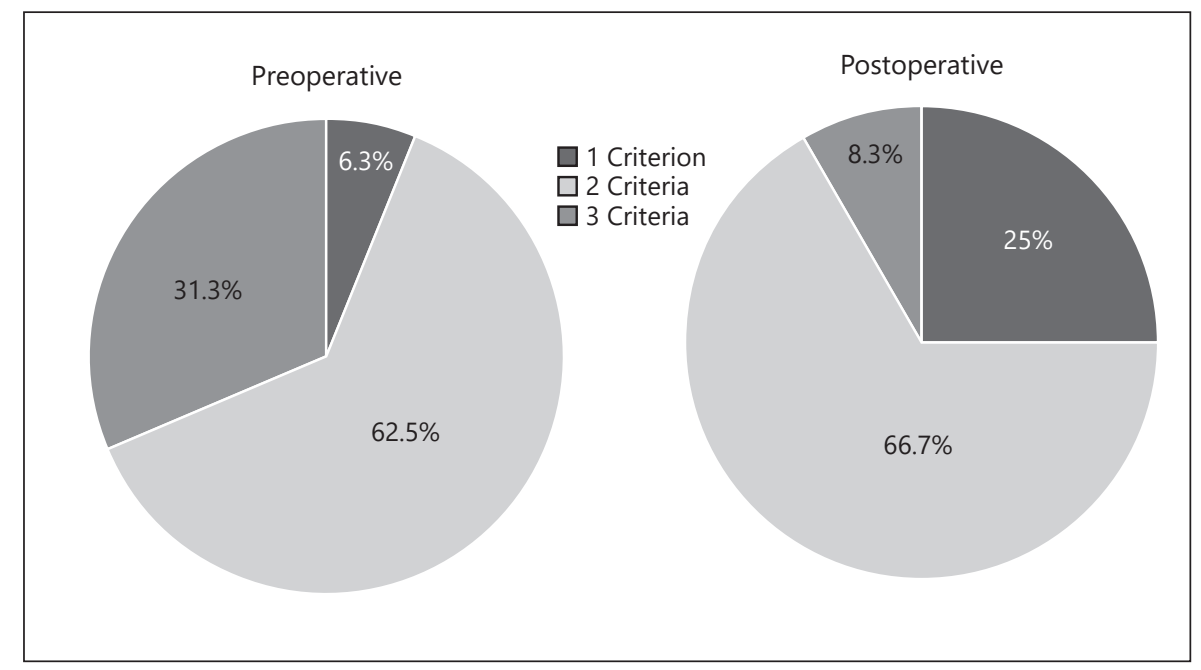

nificantly worse than preoperatively $(Z=12.161, p<$ 0.0001).

The postoperative diagnosis of PES was based on the same clinical features as the preoperative diagnosis. However, while pupillary border deposits (75\% of cases diagnosed by the second examiner) and Sampaolesi line (66.7\%) remained the cornerstones of the diagnosis, anterior capsule deposits were evident in only $41.7 \%$ of cases, which were only $31.2 \%$ of the originally diagnosed PES. Comparison of the identification of the 3 major clinical signs of PES between the 2 examiners yielded significant correlations for pupillary border deposits $\left(\chi^{2}=9.539, p<0.005\right)$ and Sampaolesi line $\left(\chi^{2}=9.332, p<0.005\right)$, but not for anterior capsular deposits $\left(\chi^{2}=6.133, p>0.05\right)$. These results are detailed in Table 2 . The sensitivity and specificity of the postoperative diagnosis of PES and each major clinical sign separately for the diagnosis of PES are shown in Figure 1.

Figure 2 demonstrates the partition of PES patients by the number of criteria found in each subject. While 5 patients $(31.2 \%)$ had 3 criteria in their examination preoperatively, only 1 patient $(8.3 \%)$ had 3 criteria postoperatively. On the other hand, only 1 patient $(6.3 \%)$ had one criterion preoperatively, compared to 3 patients (25\%) postoperatively.

\section{Discussion}

The diagnosis of PES can be based on several clinical findings, alone or in combination [1-3]. To the best of our knowledge, this is the first study to compare the diagnosis of PES before and after cataract-extraction surgery. Our findings suggest that PES might be underdiagnosed in pseudophakic patients owing to the loss of one of the most significant diagnostic sites, namely the anterior capsule of the lens. Naturally, deposits on the anterior capsule were an important diagnostic criterion for PES before surgery; however, they could not be observed postoperatively in almost $70 \%$ of the patients diagnosed with PES.

PES is a component of and a contributing factor to several ocular pathologies. Its accurate diagnosis is crucial for the appropriate evaluation and management of patients with OHT or glaucoma owing to their increased risk of deterioration, suboptimal response to several therapeutic alternatives, and poorer visual prognosis [1-3, 6-10]. PES must also be taken into consideration during management of patients with pseudophakic eyes with deteriorating visual acuity because it raises a suspicion of undiagnosed lens subluxation or dislocation $[12,13]$. Additionally, PES can be bilateral, such that its diagnosis in one eye should raise clinical suspicion and prompt a search for more subtle clinical findings in the fellow eye that could otherwise have been overlooked. This could be of utmost importance when preparing for cataract surgery in the fellow eye, since PES eyes are at greater risk for intraoperative complications, such as small pupil, shallow anterior chamber, zonule dialysis, capsule fragility, posterior capsule rent, dropped nucleus, and more [14].

In accordance with the literature, both the preoperative and postoperative diagnoses of PES in the present study were based primarily on the detection of fibrillary deposits on the pupillary border and the anterior capsule as well as the Sampaolesi line $[3,15,16]$. However, in the pseudophakic patients after partial removal of the anterior capsule, only deposits on the pupillary border and Sampaolesi line correlated with the preoperative diagnosis, making 
them the 2 crucial postoperative diagnostic signs. Moreover, it seems reasonable to assume that the second observer was less able to detect the Sampaolesi line and deposits on the pupillary border (and therefore PES in general) because of the loss of the usually very prominent anterior capsular deposits, which could have served as a leading point for the diagnosis in many cases of PES.

In the present study, the prevalence rates of the clinical criteria of PES among the affected patients were $93.8 \%$ for the Sampaolesi line, $81.3 \%$ for deposits on the anterior capsule, and $50.0 \%$ for deposits on the pupillary margin. These values are in accordance with the literature. In a study of 76 patients with PES, Wishart et al. [16] described the Sampaolesi line and pigmentation of the trabecular network in $90.8 \%$, deposits on the anterior capsule in $88.2 \%$, and deposits on the pupillary margins in $31.6 \%$.

Surprisingly, 1 patient in the present study was diagnosed with PES only postoperatively. The diagnosis was based on findings of the Sampaolesi line and deposits on the iridocorneal angle. The failure to observe these signs preoperatively could be attributed to several factors. The deposits may consist of microparticles of lens material resembling PES particles that spread in the anterior chamber as result of surgery. Alternatively, they may be inflammatory particles resembling the characteristic flakes of PES or PES particles that were possibly hidden from the first examiner but became more prominent in the angle after surgery. The increased pigmentation may have resulted from pigment dispersion during surgery. Another explanation is an interobserver discrepancy in the gonioscopic examination. Unfortunately, only histopathologic analysis may provide a definitive diagnosis. It should be noted that the Sampaolesi line is not pathognomonic for PES and is also present in pigmentary dispersion syndrome (PDS). However, the prevalence of PDS is much lower than PES, representing only $1-1.5 \%$ of openangle glaucoma cases in Caucasian countries, and therefore the Sampaolesi line is mostly met as part of PES [17].

These findings show that the importance of gonioscopy cannot be overemphasized in the search for the Sampaolesi line, as well as the importance of careful inspection of the iris border during the evaluation of OHT and glaucoma in pseudophakic patients. Unfortunately, however, while gonioscopy remains the gold standard, its limitations are well established: it is subjective, highly dependent on the examiner's skills, requires patient cooperation, and is associated with limited interobserver agreement. Furthermore, its rate of use among general ophthalmologists is low [18-21]. Although general ophthalmologists are usually less familiar and comfortable with gonioscopy, they are the ones who treat and follow most patients with OHT and glaucoma. Thus, the risk of missing PES, and consequently its inadequate treatment, may be even higher than shown in the present study.

In the current study, only 2 patients were excluded due to a complicated intra/postoperative course (1 of whom was diagnosed preoperatively with PES). However, since the prevalence of a complicated course is generally higher in PES patients, this is something that could serve as a clue for higher PES suspicion and increase the sensitivity of the postoperative test. However, if these patients are excluded from future studies, as in our case, this could potentially bias the results since these PES patients may have more advanced PES findings, making them more prone to complications but easier to diagnose.

This study has several limitations. The follow-up examination took place 1-2 weeks postoperatively. This interval may have been insufficient for new deposits to accumulate on some of the implanted intraocular lenses and remnants of the anterior capsule, potentially increasing the sensitivity of the postoperative examination. Another drawback is our restriction of the evaluation to the eye undergoing cataract surgery in order to isolate the clinical evaluation of a single eye. However, in a real-life setting, the physician would also examine the fellow eye and use it as another clue for detecting bilateral PES, which could increase the sensitivity of the diagnosis in pseudophakic patients. At the same time, our examiners were aware of the research question and they were glaucoma specialists. Hence, they were already PES-oriented during the patient examinations while probably being better at diagnosing PES than most ophthalmologists to begin with, thus achieving higher specificity than the general ophthalmologist. A different limitation stems from the fact that each patient was examined only by 2 clinicians. Turning the first examiner to the "reference standard" could be erroneous, however this is balanced by the examiner being an experienced glaucoma specialist oriented to diagnose PES. Moreover, having multiple examiners in the postoperative exam could have increased the specificity of PES diagnosis. In this case we think that having only one examiner represents the reallife situation in an appropriate way. Overall, having glaucoma specialists who feel comfortable with gonioscopy as the examiners, and specifically requesting them to diagnose PES may represent the best-case scenario for PES diagnosis in pseudophakic patients. This should be studied further, possibly by conducting future research with general ophthalmologists as the examiners. A fourth limitation results from this being a pilot study with a relatively small sample size. However, we believe that the concerns
32

Ophthalmic Res 2021;64:28-33 DOI: $10.1159 / 000508336$
Sternfeld/Luski/Sella/Zahavi/Geffen/ Pereg/Megiddo/Gaton 
raised by this study are with considerable clinical significance that encourages further research.

In conclusion, this study is the first to suggest an underdiagnosis of PES in pseudophakic patients, which has important implications for appropriate future management. To increase the rate of diagnosis, careful attention should be directed to the pupillary border, and gonioscopy should be applied on a regular basis to search for clinical factors of PES and not only for closed angle. Moreover, the documentation of PES preoperatively would also help avoid this diagnostic pitfall. Large-scale studies with longer follow-up periods are needed to corroborate our findings.

\section{Statement of Ethics}

All procedures performed in studies involving human participants were in accordance with the ethical standards of the institutional and/or national research committee and with the $1964 \mathrm{Hel}-$ sinki Declaration and its later amendments or comparable ethical standards. Each patient signed an informed consent form prior to enrolment.

\section{Disclosure Statement}

The authors have no conflicts of interest to declare.

\section{Funding Sources}

No funding was procured for this work.

\section{Author Contributions}

M.L. and N.G.: development of methodology, acquisition of data. R.S., A.Z., and E.M.: development of methodology, acquisition of data, writing, review, and/or revision of the manuscript. A.P.: development of methodology, acquisition of data, analysis and interpretation. A.S. and D.G.: conception and design, development of methodology, acquisition of data, analysis and interpretation, writing, review, and/or revision of the manuscript. All authors approved the final article.

\section{References}

1 Aboobakar IF, Johnson WM, Stamer WD, Hauser MA, Allingham RR. Major review: exfoliation syndrome; advances in disease genetics, molecular biology, and epidemiology. Exp Eye Res. 2017 Jan;154:88-103.

2 Anastasopoulos E, Founti P, Topouzis F. Update on pseudoexfoliation syndrome pathogenesis and associations with intraocular pressure, glaucoma and systemic diseases. Curr Opin Ophthalmol. 2015 Mar;26(2):829.

3 Ritch R. Exfoliation syndrome. Curr Opin Ophthalmol. 2001 Apr;12(2):124-30.

4 Aström S, Lindén C. Incidence and prevalence of pseudoexfoliation and open-angle glaucoma in northern Sweden: I. Baseline report. Acta Ophthalmol Scand. 2007 Dec; 85(8):828-31.

5 Mccarty CA, Taylor HR. Pseudoexfoliation syndrome in Australian adults. Am J Ophthalmol. 2000 May;129(5):629-33.

6 Ritch R. Exfoliation syndrome: beyond glaucoma. Arch Ophthalmol. 2008 Jun;126(6): 859-61.

7 Chan TC, Bala C, Siu A, Wan F, White A. Risk factors for rapid glaucoma disease progression. Am J Ophthalmol. 2017 Aug;180:151-7.
8 Tarkkanen AH, Kivelä TT. Comparison of primary open-angle glaucoma and exfoliation glaucoma at diagnosis. Eur J Ophthalmol. 2015 Mar-Apr;25(2):137-9.

9 Higginbotham EJ, Richardson TM. Response of exfoliation glaucoma to laser trabeculoplasty. Br J Ophthalmol. 1986 Nov;70(11): 837-9.

10 Landers J, Martin K, Sarkies N, Bourne R, Watson P. A twenty-year follow-up study of trabeculectomy: risk factors and outcomes. Ophthalmology. 2012 Apr;119(4):694-702.

11 Ritch R, Podos S. Laser trabeculoplasty in the exfoliation syndrome. Bull NY Acad Med. 1983 May;59(4):339-44.

12 Liu E, Cole S, Werner L, Hengerer F, Mamalis N, Kohnen T. Pathologic evidence of pseudoexfoliation in cases of in-the-bag intraocular lens subluxation or dislocation. J Cataract Refract Surg. 2015 May;41(5):929-35.

13 Vazquez-Ferreiro P, Carrera-Hueso FJ, FikriBenbrahim N, Barreiro-Rodriguez L, DiazRey M, Ramón Barrios MA. Intraocular lens dislocation in pseudoexfoliation: a systematic review and meta-analysis. Acta Ophthalmol. 2017 May;95(3):e164-9.

14 Shingleton BJ, Crandall AS, Ahmed II. Pseudoexfoliation and the cataract surgeon: preoperative, intraoperative, and postoperative issues related to intraocular pressure, cataract, and intraocular lenses. J Cataract Refract Surg. 2009 Jun;35(6):1101-20.
15 Prince AM, Ritch R. Clinical signs of the pseudoexfoliation syndrome. Ophthalmology. 1986 Jun;93(6):803-7.

16 Wishart PK, Spaeth GL, Poryzees EM. Anterior chamber angle in the exfoliation syndrome. Br J Ophthalmol. 1985 Feb;69(2):1037.

17 Scuderi G, Contestabile MT, Scuderi L, Librando A, Fenicia V, Rahimi S. Pigment dispersion syndrome and pigmentary glaucoma: a review and update. Int Ophthalmol. 2019 Jul;39(7):1651-62.

18 Coleman AL, Yu F, Evans SJ. Use of gonioscopy in medicare beneficiaries before glaucoma surgery. J Glaucoma. 2006 Dec;15(6):48693.

19 Salim S, Dorairaj S. Anterior segment imaging in glaucoma. Semin Ophthalmol. 2013 May;28(3):113-25.

20 Friedman DS, He M. Anterior chamber angle assessment techniques. Surv Ophthalmol. 2008 May-Jun;53(3):250-73.

21 Choudhari NS, Pathak-Ray V, Kaushik S, Vyas P, George R. Prevalent practice patterns in glaucoma: poll of Indian ophthalmologists at a national conference. Indian J Ophthalmol. 2016 Oct;64(10):715-21.
Pseudoexfoliation Syndrome in Pseudophakic Patients
Ophthalmic Res 2021;64:28-33 DOI: $10.1159 / 000508336$ 